This item was submitted to Loughborough's Research Repository by the author.

Items in Figshare are protected by copyright, with all rights reserved, unless otherwise indicated.

\title{
Water waves over arrays of horizontal cylinders: band gaps and Bragg
} resonance

PLEASE CITE THE PUBLISHED VERSION

http://dx.doi.org/10.1017/S0022112010005471

PUBLISHER

(c) Cambridge University Press

VERSION

VoR (Version of Record)

LICENCE

CC BY-NC-ND 4.0

REPOSITORY RECORD

Linton, C.M.. 2019. "Water Waves over Arrays of Horizontal Cylinders: Band Gaps and Bragg Resonance". figshare. https://hdl.handle.net/2134/15300. 
This item was submitted to Loughborough's Institutional Repository (https://dspace.lboro.ac.uk/) by the author and is made available under the following Creative Commons Licence conditions.

\section{creative
commons}

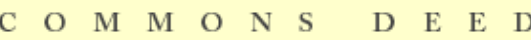

Attribution-NonCommercial-NoDerivs 2.5

You are free:

- to copy, distribute, display, and perform the work

Under the following conditions:

Attribution. You must attribute the work in the manner specified b the author or licensor.

Noncommercial. You may not use this work for commercial purposes.

No Derivative Works. You may not alter, transform, or build upon this work.

- For any reuse or distribution, you must make clear to others the license terms of this work.

- Any of these conditions can be waived if you get permission from the copyright holder.

Your fair use and other rights are in no way affected by the above.

This is a human-readable summary of the Leqal Code (the full license).

\section{Disclaimer 만}

For the full text of this licence, please go to: http://creativecommons.org/licenses/by-nc-nd/2.5/ 


\title{
Water waves over arrays of horizontal cylinders: band gaps and Bragg resonance
}

\author{
C. M. LINTON† \\ Department of Mathematical Sciences, Loughborough University, Leicestershire LE11 3TU, UK
}

(Received 4 May 2010; revised 3 September 2010; accepted 12 October 2010; first published online 25 January 2011)

The existence of a band-gap structure associated with water waves propagating over infinite periodic arrays of submerged horizontal circular cylinders in deep water is established. Waves propagating at right angles to the cylinder axes and at an oblique angle are both considered. In each case an exact linear analysis is presented with numerical results obtained by solving truncated systems of equations. Calculations for large finite arrays are also presented, which show the effect of an incident wave having a frequency within a band gap - with the amount of energy transmitted across the array tending to zero as the size of the array is increased. The location of the band gaps is not as predicted by Bragg's law, but we show that an approximate determination of their position can be made very simply if the phase of the transmission coefficient for a single cylinder is known.

Key words: wave-structure interactions

\section{Introduction}

When a plane wave is incident upon a periodic structure (periodic in the $x$-direction with period $d$, say), a resonance can occur when the component of the wavenumber in the $x$-direction is an integer multiple of $\pi / d$. This is known as Bragg's law, following William Lawrence Bragg's discovery of the phenomenon in the context of X-ray diffraction in 1912 (Hunter 2004, chapter 2). If we denote the angle that the wave makes with the $x$-axis by $\varphi$, then this is equivalent to the condition $2 d \cos \varphi=n \lambda$, where $\lambda$ is the incident wavelength. For incident waves in the $x$-direction this leads to the idea that resonances may occur when the wavelength is twice the period, with higher-order resonances for $\lambda=2 d / n, n=2,3, \ldots$

In the context of water waves (and for the purposes of this paper we exclude cases in which the depth variation can be factored out leading to an equivalent acoustics problem) Bragg resonance has been used to help explain the formation of longshore sandbars which can often be found on gently sloping beaches (see, for example, Mei, Stiassnie \& Yue 2005, chapter 7). The particular theoretical problem that has been the focus of most attention is the scattering of surface waves by a finite patch of sinusoidally varying topography in an otherwise flat ocean (see Davies 1982; Davies \& Heathershaw 1984; Mei 1985; Mei, Hara \& Naciri 1988; Chamberlain \& Porter 1995). The inclusion of an elastic plate on the free surface (modelling an ice sheet) has also been considered (Bennetts, Biggs \& Porter 2009). More recently, the effect of Bragg resonance on the efficiency of certain types of wave-energy device has been 
investigated by Garnaud \& Mei (2010). The analysis in these papers is predicated on the scatterers (bed ripples in the former case and floating buoys in the latter) being small, which then allows for some form of approximation to be made. By contrast, in this paper we consider a periodic array made up of structures whose dimensions are of a size comparable to the wavelength.

Specifically, we consider wave interaction with a periodic array of horizontal circular cylinders of radius $a$ submerged below the free surface in water of infinite depth. Everything that follows could easily be adapted to the case of constant finite depth. The undisturbed free surface is $z=0$, and the cylinder centres are at $x=j d$, $z=-f$, where $j \in \mathbb{Z}$ in the case of an infinite array or $j=0, \ldots, M-1$ if there are $M$ cylinders. Polar coordinates centred at $x=j d, z=-f$ are $\left(r_{j}, \theta_{j}\right)$ with $\theta_{j}$ measured from the downward vertical so that

$$
r_{j} \sin \theta_{j}=x-j d, \quad r_{j} \cos \theta_{j}=-z-f .
$$

We will write $(r, \theta)$ for $\left(r_{0}, \theta_{0}\right)$ where convenient. The analysis is based on linear water wave theory in which the fluid is assumed to be inviscid and incompressible and its motion assumed to be irrotational. A time factor of $\mathrm{e}^{-\mathrm{i} \omega t}$ is suppressed throughout. We assume an $\mathrm{e}^{\mathrm{i} \ell y}(\ell \geqslant 0)$ dependence for the velocity potential so that in the region $z<0, r_{j}>a$ we have to solve the two-dimensional modified Helmholtz equation $\nabla_{x z}^{2} \phi-\ell^{2} \phi=0$. The free-surface boundary condition is $\phi_{z}=K \phi$ on $z=0\left(K=\omega^{2} / g, g\right.$ being the acceleration due to gravity), and we require decay as $z \rightarrow-\infty$. The cylinders are assumed to be rigid so that $\partial \phi / \partial r_{j}=0$ on $r_{j}=a$.

The primary aim of this paper is to illustrate the link between resonant behaviour associated with scattering by finite periodic structures and the concept of stop bands in wave propagation through an infinite periodic array. The existence of ranges of frequencies for which wave propagation through a periodic array is not possible is well known and of great practical importance in a host of physical contexts. In water waves the most thorough investigations, in which the full linear problem including evanescent interactions is included, appear to be those by Chou (1998), McIver (2000) and Porter \& Porter (2003). There are many other studies, e.g. An \& Ye (2004), Chen et al. (2004) and Yang et al. (2006), in which the evanescent contributions are ignored. If an infinite periodic array has a stop band, then one would expect that this would lead to enhanced reflection when a plane wave is incident on a finite array at a frequency within such a stop band. Moreover, under such circumstances one would expect that as the size of the finite array increases, the amount of energy transmitted across the array should tend to zero.

If one assumes that the scatterers are widely spaced so that the interaction between them is governed solely by the modes propagating along the surface and not the evanescent field, then the problem is greatly simplified, and the overall effect of an array of $M$ cylinders can be determined in terms of the scattering characteristics of a single cylinder, using a transfer matrix approach. This is described briefly in $\S 2$, and it leads to a very simple condition which can be used to determine the stop and pass bands for an infinite array. One of the motivations for considering submerged circular cylinders in this context is that in the case $\ell=0$ (in deep water) the wide-spacing argument suggests that stop bands do not exist, essentially because the reflection coefficient for a single cylinder is identically zero (Dean 1948). The full linear theory, based on multipole expansions (first used for a single cylinder by Ursell 1950), is described in $\S 3$ for the case $\ell=0$ and in $\S 4$ for $\ell>0$. Numerical results are presented in $\S 5$, showing that stop bands do exist for all $\ell \geqslant 0$, leading to the possibility of 
enhanced reflection for large finite arrays when an incident wave has a frequency which lies within one of these bands.

\section{Finite array of widely spaced cylinders}

The analysis in this section is described in terms of infinite depth, but the results are equally valid for the case of constant finite depth with the appropriate change in wavenumber. We consider an array of scatterers at $x=j d, j=0, \ldots, M-1$. They need not be rigid circular cylinders; the only requirement for what follows is that they are identical. The results are equivalent (though expressed in a somewhat simpler form) to those given in Evans (1990); see also Linton \& Mclver (2001, § 6.3).

First we consider a single scatterer at $x=0$ and assume that for a wave with $x$ variation $\mathrm{e}^{\mathrm{i} \alpha x}$, where $\alpha=\left(K^{2}-\ell^{2}\right)^{1 / 2}>0$, incident from $x=-\infty$ we have the reflection and transmission coefficients $R$ and $T$, respectively. In other words, an incident wave with unit complex amplitude gives rise to a reflected (transmitted) wave with complex amplitude $R(T)$. Similarly, for a wave $\mathrm{e}^{-\mathrm{i} \alpha x}$ incident from $x=\infty$ we have the reflection and transmission coefficients $r$ and $t$, respectively. From standard linear water wave theory we have (Linton \& McIver 2001, § 1.4)

$$
t=T, \quad|r|=|R|, \quad|R|^{2}+|T|^{2}=|r|^{2}+|t|^{2}=1, \quad R \bar{t}+\bar{r} T=0 .
$$

We proceed as if these quantities are all known, though their determination will, in practice, require some numerical computation. If the scatterer is at $x=j d$, then we label the reflection and transmission coefficients as $R_{j}, r_{j}$ and $T_{j}, t_{j}$, respectively, and

$$
T_{j}=t_{j}=T, \quad R_{j}=R \mathrm{e}^{2 \mathrm{i} j \alpha d}, \quad r_{j}=r \mathrm{e}^{-2 \mathrm{i} j \alpha d} .
$$

For a single scatterer at $x=j d$ we define the transfer matrix $\boldsymbol{S}_{j}$ by

$$
\left(\begin{array}{c}
A_{j+1} \\
B_{j+1}
\end{array}\right)=\boldsymbol{S}_{j}\left(\begin{array}{c}
A_{j} \\
B_{j}
\end{array}\right),
$$

which relates the amplitudes of the waves to the right of the scatterer $\left(A_{j+1}\right.$ propagating to the right and $B_{j+1}$ to the left) to those on the left ( $A_{j}$ propagating to the right and $B_{j}$ to the left). The definitions of the reflection and transmission coefficients imply that

from which

$$
\left(\begin{array}{cc}
T_{j} & r_{j} \\
0 & 1
\end{array}\right)=\boldsymbol{S}_{j}\left(\begin{array}{cc}
1 & 0 \\
R_{j} & t_{j}
\end{array}\right)
$$

$$
\boldsymbol{S}_{j}=\frac{1}{t_{j}}\left(\begin{array}{cc}
T_{j} t_{j}-R_{j} r_{j} & r_{j} \\
-R_{j} & 1
\end{array}\right)=\left(\begin{array}{cc}
1 / \bar{t} & (r / t) \mathrm{e}^{-2 \mathrm{i} j \alpha d} \\
(\overline{r / t}) \mathrm{e}^{2 \mathrm{i} j \alpha d} & 1 / t
\end{array}\right)
$$

on use of (2.1) and (2.2). If we write $r=\rho \mathrm{e}^{2 \mathrm{i} \delta_{r}}$ and $t=\tau \mathrm{e}^{2 \mathrm{i} \delta_{t}}$ we obtain

$$
\boldsymbol{S}_{j}=\left(\begin{array}{cc}
\mathrm{e}^{\mathrm{i} \alpha_{j}} & 0 \\
0 & \mathrm{e}^{-\mathrm{i} \alpha_{j}}
\end{array}\right)\left(\begin{array}{cc}
1 / \tau & \rho / \tau \\
\rho / \tau & 1 / \tau
\end{array}\right)\left(\begin{array}{cc}
\mathrm{e}^{\mathrm{i} \beta_{j}} & 0 \\
0 & \mathrm{e}^{-\mathrm{i} \beta_{j}}
\end{array}\right)=\boldsymbol{A}_{j} \boldsymbol{R} \boldsymbol{B}_{j},
$$

say, where

$$
\alpha_{j}=\delta_{r}-j \alpha d, \quad \beta_{j}=2 \delta_{t}-\delta_{r}+j \alpha d .
$$

For an array of scatterers at $x=j d, j=0, \ldots, M-1$ and an incident wave from the left with $x$-dependence $\mathrm{e}^{\mathrm{i} \alpha x}$, we denote the overall reflection and transmission 
coefficients by $R_{M}$ and $T_{M}$, respectively. If we treat the scatterers as widely separated so that multiple scattering effects are limited to the propagating waves, then we have

$$
\left(\begin{array}{c}
T_{M} \\
0
\end{array}\right)=\boldsymbol{A}_{M-1} \boldsymbol{R B}_{M-1} \boldsymbol{A}_{M-2} \boldsymbol{R} \boldsymbol{B}_{M-2} \ldots \boldsymbol{A}_{1} \boldsymbol{R} \boldsymbol{B}_{1} \boldsymbol{A}_{0} \boldsymbol{R} \boldsymbol{B}_{0}\left(\begin{array}{c}
1 \\
R_{M}
\end{array}\right) .
$$

Let $\chi=\beta_{j+1}+\alpha_{j}=2 \delta_{t}+\alpha d$, which is independent of $j$. Then

$$
\boldsymbol{B}_{j+1} \boldsymbol{A}_{j} \boldsymbol{R}=\left(\begin{array}{cc}
\mathrm{e}^{\mathrm{i} \chi} / \tau & \rho \mathrm{e}^{\mathrm{i} \chi} / \tau \\
\rho \mathrm{e}^{-\mathrm{i} \chi} / \tau & \mathrm{e}^{-\mathrm{i} \chi} / \tau
\end{array}\right)=\boldsymbol{W}, \quad \text { say, }
$$

and

$$
\left(\begin{array}{c}
T_{M} \\
0
\end{array}\right)=\boldsymbol{B}_{M}^{-1} \boldsymbol{W}^{M} \boldsymbol{B}_{0}\left(\begin{array}{c}
1 \\
R_{M}
\end{array}\right)
$$

Sicne $\rho^{2}+\tau^{2}=1$, it is clear that $\operatorname{det} \boldsymbol{W}=1$ and trace $\boldsymbol{W}=(2 / \tau) \cos \chi$ which is real. Hence the eigenvalues of $\boldsymbol{W}$ are

$$
\lambda_{1}=\mathrm{e}^{\mathrm{i} q} \quad \text { and } \quad \lambda_{2}=\mathrm{e}^{-\mathrm{i} q},
$$

where $\cos q=(1 / \tau) \cos \chi$ and $q$ will be real or complex depending on whether $\tau>|\cos \chi|$ or $\tau<|\cos \chi|$. (Note that $q=\pi+$ iy, with $y$ real, if $\cos \chi<-1$.) We then have (Markoš \& Soukoulis 2008, § 1.5.2)

$$
\boldsymbol{W}^{n}=\left(\begin{array}{cc}
(1 / \tau) \mathrm{e}^{\mathrm{i} \chi} U_{n-1}-U_{n-2} & (\rho / \tau) \mathrm{e}^{\mathrm{i} \chi} U_{n-1} \\
(\rho / \tau) \mathrm{e}^{-\mathrm{i} \chi} U_{n-1} & (1 / \tau) \mathrm{e}^{-\mathrm{i} \chi} U_{n-1}-U_{n-2}
\end{array}\right),
$$

where

$$
U_{n} \equiv U_{n}(\cos q)=\frac{\sin (n+1) q}{\sin q}
$$

is a Chebyshev polynomial of the second kind. It can then be shown, from (2.10), that

$$
T_{M}=\frac{\tau \mathrm{e}^{-\mathrm{i} M \alpha d}}{\mathrm{e}^{-\mathrm{i} \chi} U_{M-1}-\tau U_{M-2}},
$$

and a straightforward calculation (using the identities $U_{n-2}-2 \cos q U_{n-1}+U_{n}=0$ and $U_{n} U_{n-2}=U_{n-1}^{2}-1$ ) reveals that

$$
\left|T_{M}\right|^{2}=\frac{\tau^{2}}{\tau^{2}+\rho^{2} U_{M-1}^{2}} .
$$

If $q$ is real, then clearly $\left|T_{M}\right|^{2}$ oscillates as $M$ varies. (It is still possible to take the limit of $R_{M}$ as $M \rightarrow \infty$ by introducing some artificial damping first; see Porter \& Evans 2006). However, if $q$ is complex, which happens if

$$
\tau<|\cos \chi|
$$

then $\left|T_{M}\right| \rightarrow 0$ as $M \rightarrow \infty$. Hence, in the context of water waves, (2.16) is the condition for a stop band in an infinite array of widely spaced scatterers (a result which appears to have been given first by McIver 2000). Some general conclusions follow from (2.16). The smaller the value of $\tau$ is (i.e. the stronger the individual elements of the array are at reflecting waves), the larger the band gaps are likely to be, and one would expect the band gaps to be centred around the points $\chi=n \pi$. If the phase of the transmission coefficient is small, then these points are approximately $\alpha d=n \pi$, which is Bragg's law. 
The fact that band gaps are sometimes shifted away from the Bragg values has been observed previously in related problems; e.g. see Porter \& Porter 2003 and Bennetts et al. 2009, and the former of these papers provides an alternative explanation for this drift. A classic example of a scatterer for which $\delta_{t}$ is not negligible even though it is very poor at reflecting waves is a submerged horizontal circular cylinder in deep water, for which $\rho \equiv 0$ if $\ell=0$, and the rest of the paper deals with arrays of such scatterers.

It is possible to extend the transfer matrix approach to include evanescent wave interactions (Devillard, Dunlop \& Souillard 1988), and this has been done in the context of periodic bed topographies in Porter \& Porter (2003). Here we take a different approach to solving the full linear problem.

\section{Full linear theory: waves perpendicular to the cylinders}

When $\ell=0$ the modified Helmholtz equation reduces to Laplace's equation. In this case, the ingredients for treating an array of submerged circular cylinders through mutipole expansions can be found in O'Leary (1985) and Linton \& McIver (2001, $\S 3.2$ ). Multipoles singular at $r_{j}=0$ and symmetric in $\theta_{j}$ are (Linton \& McIver 2001, (B.17))

$$
\phi_{n}^{j}=\frac{\cos n \theta_{j}}{r_{j}^{n}}+\frac{(-1)^{n}}{(n-1) !} f_{0}^{\infty} \frac{\mu+K}{\mu-K} \mu^{n-1} \exp [\mu(z-f)] \cos \mu(x-j d) \mathrm{d} \mu, \quad n \geqslant 1,
$$

and multipoles singular at $r_{j}=0$ and antisymmetric in $\theta_{j}$ are (Linton \& McIver 2001, (B.26))

$$
\psi_{n}^{j}=\frac{\sin n \theta_{j}}{r_{j}^{n}}+\frac{(-1)^{n+1}}{(n-1) !} f_{0}^{\infty} \frac{\mu+K}{\mu-K} \mu^{n-1} \exp [\mu(z-f)] \sin \mu(x-j d) \mathrm{d} \mu, \quad n \geqslant 1 .
$$

The integrals here are contour integrals indented so as to pass beneath the poles that exist on the real axis at $\mu=K$, which ensures that they behave like outgoing waves as $|x| \rightarrow \infty$. For $z>-f$ we also have (Linton \& McIver 2001, (B.3))

$$
\phi_{n}^{j}=\frac{2(-1)^{n}}{(n-1) !} f_{0}^{\infty} \frac{\mu^{n-1} \mathrm{e}^{-\mu f}}{\mu-K}(\mu \cosh \mu z+K \sinh \mu z) \cos \mu(x-j d) \mathrm{d} \mu,
$$

and for $\psi_{n}^{j}$ one simply replaces cos by $-\sin$ in this expression. Derivations of these formulae can be found in Thorne (1953).

We can develop series expansions for these multipoles about the point $x=k d$, $z=-f$. Thus, for all $j, k$ and for $r_{k}<2 f$,

$$
\begin{aligned}
& \phi_{n}^{j}=\frac{\cos n \theta_{j}}{r_{j}^{n}}+\sum_{m=0}^{\infty} P_{m n}^{j k} r_{k}^{m} \cos m \theta_{k}-\sum_{m=1}^{\infty} Q_{m n}^{j k} r_{k}^{m} \sin m \theta_{k}, \\
& \psi_{n}^{j}=\frac{\sin n \theta_{j}}{r_{j}^{n}}+\sum_{m=0}^{\infty} Q_{m n}^{j k} r_{k}^{m} \cos m \theta_{k}+\sum_{m=1}^{\infty} P_{m n}^{j k} r_{k}^{m} \sin m \theta_{k},
\end{aligned}
$$

where

$$
\left\{\begin{array}{c}
P_{m n}^{j k} \\
Q_{m n}^{j k}
\end{array}\right\}=\frac{(-1)^{m+n}}{m !(n-1) !} f_{0}^{\infty} \frac{\mu+K}{\mu-K} \mu^{m+n-1} \mathrm{e}^{-2 \mu f}\left\{\begin{array}{c}
\cos \mu(j-k) d \\
\sin \mu(j-k) d
\end{array}\right\} \mathrm{d} \mu
$$


in an obvious notation. For computational purposes we note that the integral in (3.6) can be written as the sum of a principal-value integral and a contribution from the residue at the pole. This can be manipulated to yield

$$
\begin{aligned}
a^{m+n} P_{m n}^{j k}= & \frac{(-1)^{m+n}}{m !(n-1) !}\left[\int _ { 0 } ^ { 2 K a } \left((u+K a) u^{m+n-1} \mathrm{e}^{-2 u f / a} \cos u(j-k) d / a\right.\right. \\
& \left.-2(K a)^{m+n} \mathrm{e}^{-2 K f} \cos K d(j-k)\right) \frac{\mathrm{d} u}{u-K a} \\
& +\int_{2 K a}^{\infty} \frac{u+K a}{u-K a} u^{m+n-1} \mathrm{e}^{-2 u f / a} \cos u(j-k) d / a \mathrm{~d} u \\
& \left.+2 \pi \mathrm{i}(K a)^{m+n} \mathrm{e}^{-2 K f} \cos K d(j-k)\right],
\end{aligned}
$$

where we have evaluated the principal-value integral

$$
f_{0}^{2 K a} \frac{\mathrm{d} u}{u-K a}=0 .
$$

Similarly for $Q_{m n}^{j k}$, just replace cos by sin.

To re-expand the singular terms about $x=k d, z=-f$ we use the technique described in Linton \& McIver $(2001, \S 3.2 .1)$; see also the Appendix to O'Leary (1985). We can thus show that for $j \neq k, r_{k}<\min (2 f,|j-k| d)$,

$$
\begin{aligned}
& \phi_{n}^{j}=\sum_{m=0}^{\infty}\left(P_{m n}^{j k}+C_{m n}^{j k}\right) r_{k}^{m} \cos m \theta_{k}-\sum_{m=1}^{\infty}\left(Q_{m n}^{j k}+S_{m n}^{j k}\right) r_{k}^{m} \sin m \theta_{k}, \\
& \psi_{n}^{j}=\sum_{m=0}^{\infty}\left(Q_{m n}^{j k}-S_{m n}^{j k}\right) r_{k}^{m} \cos m \theta_{k}+\sum_{m=1}^{\infty}\left(P_{m n}^{j k}-C_{m n}^{j k}\right) r_{k}^{m} \sin m \theta_{k},
\end{aligned}
$$

where

$$
\left\{\begin{array}{c}
C_{m n}^{j k} \\
S_{m n}^{j k}
\end{array}\right\}=\frac{(n+m-1) !}{((j-k) d)^{n+m} m !(n-1) !}\left\{\begin{array}{l}
\cos \left[(n-m) \frac{\pi}{2}\right] \\
\sin \left[(n-m) \frac{\pi}{2}\right]
\end{array}\right\} .
$$

\subsection{Infinite array}

The case of an infinite array of identical submerged circular cylinders has been treated previously by Schnute (1967) using an integral equation formulation as in Levine (1965). The method presented below is much simpler than that given in Schnute's work. We seek a solution in the form

$$
\phi=\sum_{j=-\infty}^{\infty} \mathrm{e}^{\mathrm{i} \beta j d} \sum_{n=1}^{\infty} a^{n}\left(\xi_{n} \phi_{n}^{j}+\eta_{n} \psi_{n}^{j}\right),
$$

where, without loss of generality, we can assume that $0 \leqslant \beta d<2 \pi$, and $\xi_{n}$ and $\eta_{n}$ are complex unknowns that we need to determine. It is straightforward to show that $\phi(x+d, z)=\mathrm{e}^{\mathrm{i} \beta d} \phi(x, z)$, and therefore $\phi$ is quasi-periodic. Because of the symmetry of the geometry about $x=0$ we can also show that if there is a non-trivial solution for a particular value of $\beta d$, there will also be a solution with $\beta d$ replaced by $2 \pi-\beta d$ corresponding to a wave travelling in the opposite direction. Hence we can restrict attention to $0 \leqslant \beta d \leqslant \pi$. 
On the assumption that there is a solution of this form, from (3.3), for $z>-f$,

$$
\begin{aligned}
\phi=\sum_{n=1}^{\infty} & \frac{a^{n}(-1)^{n}}{(n-1) !} \sum_{j=-\infty}^{\infty} \exp (\mathrm{i} \beta j d) \int_{0}^{\infty} \frac{\mu^{n-1} \exp (-\mu f)}{\mu-K}(\mu \cosh \mu z+K \sinh \mu z) \\
& \times\left(\left(\xi_{n}+\mathrm{i} \eta_{n}\right) \exp [\mathrm{i} \mu(x-j d)]+\left(\xi_{n}-\mathrm{i} \eta_{n}\right) \exp [-\mathrm{i} \mu(x-j d)]\right) \mathrm{d} \mu,
\end{aligned}
$$

which after application of the Poisson summation formula, writing $\beta_{j}=\beta+2 j \pi / d$, yields

$$
\phi=\sum_{j=-\infty}^{\infty} \mathscr{A}_{j} \frac{\left(\left|\beta_{j}\right| \cosh \beta_{j} z+K \sinh \left|\beta_{j}\right| z\right)}{\left|\beta_{j}\right|-K} \exp \left(\mathrm{i} \beta_{j} x-\left|\beta_{j}\right| f\right)
$$

where

$$
\mathscr{A}_{j}=\sum_{n=1}^{\infty} \frac{2 \pi a^{n}(-1)^{n}}{d(n-1) !}\left|\beta_{j}\right|^{n-1}\left(\xi_{n}+\operatorname{sgn}\left(\beta_{j}\right) i \eta_{n}\right) .
$$

This requires that we assume $\left|\beta_{j}\right| \neq K$ for any $j$. However, we will see later that there is no singularity when this occurs because $\mathscr{A}_{j}$ then vanishes. The solution can thus be represented as an infinite sum of modes, each having a different wavenumber and a different amplitude. The free-surface elevation, which is given by $\left.(\mathrm{i} \omega / g) \phi\right|_{z=0}$, can be obtained from (3.14).

The form of the potential given by (3.12) satisfies all the conditions of the problem except the boundary condition on the cylinders. In fact, we need to satisfy only the boundary condition on one cylinder (which we take to be the one at the origin), and then all the others are satisfied because of quasi-periodicity. If we write

$$
\left\{\begin{array}{l}
\hat{P}_{m n} \\
\hat{Q}_{m n}
\end{array}\right\}=a^{n+m} \sum_{j=-\infty}^{\infty} \mathrm{e}^{\mathrm{i} \beta j d}\left\{\begin{array}{l}
P_{m n}^{j 0} \\
Q_{m n}^{j 0}
\end{array}\right\}, \quad\left\{\begin{array}{l}
\hat{C}_{m n} \\
\hat{S}_{m n}
\end{array}\right\}=a^{n+m} \sum_{j=-\infty}^{\infty} \mathrm{e}^{\mathrm{i} \beta j d}\left\{\begin{array}{l}
C_{m n}^{j 0} \\
S_{m n}^{j 0}
\end{array}\right\}
$$

(the $j=0$ term is included in the definition of $\hat{Q}_{m n}$ even though it is zero, and the dash on the summation indicates that the $j=0$ term should be omitted), then setting $\partial \phi / \partial r=0$ on $r=a$ yields

$$
\begin{aligned}
& \xi_{m}-\sum_{n=1}^{\infty}\left(\xi_{n}\left(\hat{P}_{m n}+\hat{C}_{m n}\right)+\eta_{n}\left(\hat{Q}_{m n}-\hat{S}_{m n}\right)\right)=0, \quad m \geqslant 1, \\
& \eta_{m}+\sum_{n=1}^{\infty}\left(\xi_{n}\left(\hat{Q}_{m n}+\hat{S}_{m n}\right)-\eta_{n}\left(\hat{P}_{m n}-\hat{C}_{m n}\right)\right)=0, \quad m \geqslant 1 .
\end{aligned}
$$

These equations will later be used, in truncated form, to determine numerical approximations to $\xi_{n}$ and $\eta_{n}$.

The quantities $\hat{P}_{m n}, \hat{Q}_{m n}, \hat{C}_{m n}$ and $\hat{S}_{m n}$ can all be evaluated simply. In the case of $\hat{C}_{m n}$ and $\hat{S}_{m n}$ the series in (3.16) can actually be summed explicitly. If $n+m$ is odd, then $\hat{C}_{m n}=0$. On the other hand, if $n+m$ is even $(\geqslant 2)$ we have

$$
\hat{C}_{m n}=\frac{(-1)^{n+1}(2 \pi a / d)^{n+m}}{m !(n-1) !(n+m)} \mathscr{B}_{n+m}(\beta d / 2 \pi),
$$

where $\mathscr{B}_{n}(\cdot)$ is a Bernoulli polynomial and we have used Abramowitz \& Stegun (1965, (23.1.18)). This is valid for all $\beta d$ in the interval $[0,2 \pi]$ and hence for all $\beta d$ under consideration here. Similarly if $n+m$ is even, then $\hat{S}_{m n}=0$, whereas if $n+m$ is odd 
$(\geqslant 1)$ we have

$$
\hat{S}_{m n}=\frac{\mathrm{i}(-1)^{n}(2 \pi a / d)^{n+m}}{m !(n-1) !(n+m)} \mathscr{B}_{n+m}(\beta d / 2 \pi)
$$

(Abramowitz \& Stegun 1965, (23.1.17)). Equation (3.20) is not valid when $m+n=1$ and $\beta d=0$, but this case is covered by the fact that $\hat{S}_{m n}=0$ when $\beta d=0$ or $\pi$, and (3.20) is valid for all other $\beta d$ in the interval $(0,2 \pi)$. The fact that $\hat{C}_{m n}$ and $\hat{S}_{m n}$ can be expressed so simply is a particularly attractive feature of the solution procedure.

Next $\hat{P}_{m n}$ and $\hat{Q}_{m n}$ can be expressed as exponentially convergent series, using the Poisson summation formula. We obtain

$$
\begin{aligned}
& \hat{P}_{m n}=\frac{(-a / d)^{m+n} \pi}{m !(n-1) !} \sum_{j=-\infty}^{\infty} \frac{\left|\beta_{j}\right|+K}{\left|\beta_{j}\right|-K}\left|\beta_{j} d\right|^{m+n-1} \mathrm{e}^{-2\left|\beta_{j}\right| f}, \\
& \hat{Q}_{m n}=\frac{(-a / d)^{m+n} \pi \mathrm{i}}{m !(n-1) !} \sum_{j=-\infty}^{\infty} \operatorname{sgn}\left(\beta_{j}\right) \frac{\left|\beta_{j}\right|+K}{\left|\beta_{j}\right|-K}\left|\beta_{j} d\right|^{m+n-1} \mathrm{e}^{-2\left|\beta_{j}\right| f} .
\end{aligned}
$$

It is worth noting that the contribution from the pole at $\mu=K$ plays no role, and this is consistent with the fact that the radiation condition which stipulates that the contour must pass beneath the pole is no longer relevant when there is an infinite array extending to $x= \pm \infty$. In deriving (3.21) and (3.22) we have to assume that $\left|\beta_{j}\right| \neq K$ for any $j$. However, as $\left|\beta_{j}\right| \rightarrow K$ for a particular $j, \hat{P}_{m n}$ and $\hat{Q}_{m n}$ clearly dominate in (3.17) and (3.18), and if we substitute the leading-order behaviour from (3.21) and (3.22) (i.e. retain only the singular terms) it is straightforward to show that (3.17) and (3.18) each reduce to

$$
\xi_{n}+\mathrm{i} \operatorname{sgn}\left(\beta_{j}\right) \eta_{n}=0, \quad n \geqslant 1,
$$

which shows that $\mathscr{A}_{j}$, given by (3.15), is then zero.

To formulate (3.17) and (3.18) as a finite linear system we write

$$
\left(x_{1}, x_{2}, \ldots, x_{2 N}\right)=\left(\xi_{1}, \mathrm{i} \eta_{1}, \xi_{2}, \mathrm{i} \eta_{2}, \ldots, \xi_{N}, \mathrm{i} \eta_{N}\right) .
$$

In other words $\xi_{m}=x_{2 m-1}$ and $\eta_{m}=-\mathrm{i} x_{2 m}$. Then (3.17) and (3.18) become

$$
\begin{array}{r}
x_{2 m-1}-\sum_{n=1}^{N}\left(x_{2 n-1}\left(\hat{P}_{m n}+\hat{C}_{m n}\right)-\mathrm{i} x_{2 n}\left(\hat{Q}_{m n}-\hat{S}_{m n}\right)\right)=0, \quad 1 \leqslant m \leqslant N, \\
x_{2 m}+\sum_{n=1}^{N}\left(\mathrm{i} x_{2 n-1}\left(\hat{Q}_{m n}+\hat{S}_{m n}\right)-x_{2 n}\left(\hat{P}_{m n}-\hat{C}_{m n}\right)\right)=0, \quad 1 \leqslant m \leqslant N,
\end{array}
$$

and therefore we require the determinant of the $2 N \times 2 N$ matrix $\boldsymbol{M}=\left(M_{i j}\right)_{i, j=1}^{2 N}$ to vanish, where

$$
\left.\begin{array}{rl}
M_{2 m-1,2 n-1} & =\delta_{m n}-\hat{P}_{m n}-\hat{C}_{m n}, \quad M_{2 m-1,2 n}=\mathrm{i} \hat{Q}_{m n}-\mathrm{i} \hat{S}_{m n}, \\
M_{2 m, 2 n-1} & =\mathrm{i} \hat{Q}_{m n}+\mathrm{i} \hat{S}_{m n}, \quad M_{2 m, 2 n}=\delta_{m n}-\hat{P}_{m n}+\hat{C}_{m n},
\end{array}\right\}
$$

in which all the entries are real. Numerical solutions to these equations are presented in $\S 5$. 
We now seek a solution in the form

$$
\phi=\phi_{i n c}+\phi_{s c}=\phi_{i n c}+\sum_{j=0}^{M-1} \sum_{n=1}^{\infty} a^{n}\left(\xi_{n}^{j} \phi_{n}^{j}+\eta_{n}^{j} \psi_{n}^{j}\right),
$$

in which

$$
\phi_{i n c}=\mathrm{e}^{\mathrm{i} K x} \mathrm{e}^{K z}=\mathrm{e}^{\mathrm{i} k K d} \mathrm{e}^{-K f} \sum_{n=0}^{\infty} \frac{\left(-K r_{k}\right)^{n}}{n !} \mathrm{e}^{-\mathrm{i} n \theta_{k}} .
$$

Re-expanding about $r_{k}=0$ and then setting $\partial \phi / \partial r_{k}=0$ on $r_{k}=a$ give rise to

$$
\begin{aligned}
& \xi_{m}^{k}-\sum_{n=1}^{\infty} \sum_{j=0}^{M-1} a^{m+n}\left(\xi_{n}^{j}\left(P_{m n}^{j k}+C_{m n}^{j k}\right)+\eta_{n}^{j}\left(Q_{m n}^{j k}-S_{m n}^{j k}\right)\right)=\mathrm{e}^{\mathrm{i} k K d} \mathrm{e}^{-K f} \frac{(-K a)^{m}}{m !}, \\
& \eta_{m}^{k}+\sum_{n=1}^{\infty} \sum_{j=0}^{M-1} a^{m+n}\left(\xi_{n}^{j}\left(Q_{m n}^{j k}+S_{m n}^{j k}\right)-\eta_{n}^{j}\left(P_{m n}^{j k}-C_{m n}^{j k}\right)\right)=-\mathrm{i} \mathrm{e}^{\mathrm{i} k K d} \mathrm{e}^{-K f} \frac{(-K a)^{m}}{m !},
\end{aligned}
$$

with $m \geqslant 1, k=0, \ldots, M-1$ in each case. For convenience, we have defined $C_{m n}^{k k}=S_{m n}^{k k}=0$. This can be truncated easily by restricting $m$ and $n$ to range between 1 and $N$, which yields a $2 M N \times 2 M N$ system of equations. Again, numerical solutions to these equations are presented in $\S 5$.

From (3.28) and Linton \& McIver (2001, (B.18) and (B.27)), we have that as $x \rightarrow \pm \infty$,

$$
\phi \sim \mathrm{e}^{\mathrm{i} K x} \mathrm{e}^{K z}+2 \pi \exp ( \pm \mathrm{i} K x) \exp [K(z-f)] \sum_{j=0}^{M-1} \sum_{n=1}^{\infty} \frac{(-K a)^{n}}{(n-1) !} \exp (\mp \mathrm{i} K j d)\left(\mathrm{i} \xi_{n}^{j} \mp \eta_{n}^{j}\right) .
$$

Hence the reflection and transmission coefficients are

$$
\begin{aligned}
R_{M} & =2 \pi \mathrm{e}^{-K f} \sum_{j=0}^{M-1} \sum_{n=1}^{\infty} \frac{(-K a)^{n}}{(n-1) !} \mathrm{e}^{\mathrm{i} K j d}\left(\mathrm{i} \xi_{n}^{j}+\eta_{n}^{j}\right), \\
T_{M} & =1+2 \pi \mathrm{e}^{-K f} \sum_{j=0}^{M-1} \sum_{n=1}^{\infty} \frac{(-K a)^{n}}{(n-1) !} \mathrm{e}^{-\mathrm{i} K j d}\left(\mathrm{i} \xi_{n}^{j}-\eta_{n}^{j}\right) .
\end{aligned}
$$

Alternative expressions for these coefficients can be obtained by determining the horizontal and vertical exciting forces on the cylinders and then using standard reciprocity relations (as described in, for example, Newman 1975 or Linton \& McIver $2001, \S 1.4)$. The array is not symmetric about $x=0$, but if this is accounted for we can show that

$$
R_{M}=\frac{1}{2}(Z / \bar{Z}+X / \bar{X}), \quad T_{M}=\frac{1}{2} \exp [-\mathrm{i}(M-1) K d](Z / \bar{Z}-X / \bar{X}),
$$

where we have written

$$
X=\sum_{j=0}^{M-1} \eta_{1}^{j}, \quad Z=\sum_{j=0}^{M-1} \xi_{1}^{j} .
$$

The quantities $X$ and $Z$ are proportional to, respectively, the horizontal and vertical exciting forces on the cylinder array. To see this, note that the horizontal force on 
cylinder $k$ is proportional to

$$
\int_{r_{k}=a} \phi \sin \theta_{k} \mathrm{~d} \theta_{k}=2 \pi \eta_{1}^{k}
$$

the integral being evaluated by substituting for $\phi$ from (3.28), suitably re-expanded about $r_{k}=0$, and then using (3.31) with $m=1$. Similarly for the vertical force, replace $\sin \theta_{k}$ by $-\cos \theta_{k}$, and then the integral evaluates to $-2 \pi \xi_{1}^{k}$.

For a single cylinder, it follows from (3.33) that $R_{1}=0$, since in that case (3.30) and (3.31) yield $\eta_{m}^{0}=-\mathrm{i} \xi_{m}^{0}$ for all $m$. The fact that in general $R_{M} \neq 0$ for $M>1$ is hardly surprising, though this seems to have been established first in Schnute (1971), where the case $M=2$ was considered. An analysis based on matched asymptotic expansions was performed in McIver (1990) under the assumption that the wavelength is much greater than the cylinder spacing which in turn is much greater than the cylinder radius, leading to the conclusion that $R_{M}=O\left((K d)^{2}(a / d)^{4}\right)$ in this limit.

\section{Full linear theory: oblique waves}

Multipole expansions have been used previously to solve scattering problems involving oblique wave interactions with finite arrays of submerged, horizontal circular cylinders in Shen \& Zheng (2007). In fact that paper considered the case of finite water depth. Nevertheless much of the detail remains the same, and only brief details will be given here, focusing on the infinite array case.

In the case of a finite array we will take as our incident field a plane wave making an angle $\varphi \in(0, \pi / 2)$ with the positive $x$-axis, given by

$$
\phi_{i n c}=\mathrm{e}^{\mathrm{i} \alpha x} \mathrm{e}^{K z}
$$

(having suppressed the $\mathrm{e}^{\mathrm{i} \ell y}$ dependence), where

$$
\alpha=K \cos \varphi, \quad \ell=K \sin \varphi .
$$

In particular, we note that in this case $0<\ell<K$. However, for an infinite array there is no such upper limit for $\ell$. For a finite array we could also consider the case of $\ell>K$ with no incident wave and look for trapped modes, as in Porter \& Evans (1998).

Multipoles singular at $r_{j}=0$ and symmetric in $\theta_{j}$ are now (Linton \& McIver 2001, (B.111))

$$
\begin{aligned}
\phi_{n}^{j}=\mathrm{K}_{n}\left(\ell r_{j}\right) \cos n \theta_{j}+(-1)^{n} f_{0}^{\infty} \frac{v+K}{v-K} \cosh n \mu \cos (\lambda(x-j d)) & \\
& \times \exp [v(z-f)] \mathrm{d} \mu, \quad n \geqslant 0,
\end{aligned}
$$

where $\nu=\ell \cosh \mu$ and $\lambda=\ell \sinh \mu$, and multipoles singular at $r_{j}=0$ and antisymmetric in $\theta_{j}$ are (Linton \& McIver 2001, (B.117))

$$
\begin{aligned}
\psi_{n}^{j}=\mathrm{K}_{n}\left(\ell r_{j}\right) \sin n \theta_{j}-(-1)^{n} f_{0}^{\infty} \frac{v+K}{v-K} \sinh n \mu \sin (\lambda(x-j d)) & \\
& \times \exp [v(z-f)] \mathrm{d} \mu, \quad n \geqslant 1 .
\end{aligned}
$$

Here $\mathrm{K}_{n}(\cdot)$ (and $\mathrm{I}_{n}(\cdot)$ which appears below) are modified Bessel functions. For $z>-f$ we also have (Linton \& McIver 2001, (B.101))

$$
\phi_{n}^{j}=2(-1)^{n} f_{0}^{\infty} \frac{\mathrm{e}^{-v f}}{v-K}(v \cosh v z+K \sinh v z) \cosh n \mu \cos (\lambda(x-j d)) \mathrm{d} \mu,
$$


with $\psi_{n}^{j}$ given by the same expression after replacing $\cosh n \mu$ by $\sinh n \mu$ and $\cos$ by - sin. The method by which these multipoles are derived is described in (Ursell 1951, Note A).

As before we can develop series expansions for these multipoles about the point $x=k d, z=-f$. For all $j, k$ we have, for $r<2 f$,

$$
\begin{aligned}
& \phi_{n}^{j}=\mathrm{K}_{n}\left(\ell r_{j}\right) \cos n \theta_{j}+\sum_{m=0}^{\infty} P_{m n}^{j k} \mathrm{I}_{m}\left(\ell r_{k}\right) \cos m \theta_{k}-\sum_{m=1}^{\infty} Q_{m n}^{j k} \mathrm{I}_{m}\left(\ell r_{k}\right) \sin m \theta_{k}, \\
& \psi_{n}^{j}=\mathrm{K}_{n}\left(\ell r_{j}\right) \sin n \theta_{j}+\sum_{m=0}^{\infty} R_{m n}^{j k} \mathrm{I}_{m}\left(\ell r_{k}\right) \cos m \theta_{k}+\sum_{m=1}^{\infty} S_{m n}^{j k} \mathrm{I}_{m}\left(\ell r_{k}\right) \sin m \theta_{k},
\end{aligned}
$$

where

$$
\begin{aligned}
P_{m n}^{j k} & =\epsilon_{m}(-1)^{m+n} f_{0}^{\infty} \frac{v+K}{v-K} \cosh m \mu \cosh n \mu \cos [\lambda d(j-k)] \mathrm{e}^{-2 v f} \mathrm{~d} \mu, \\
Q_{m n}^{j k} & =\frac{2}{\epsilon_{n}} R_{n m}^{j k}=2(-1)^{m+n} f_{0}^{\infty} \frac{v+K}{v-K} \sinh m \mu \cosh n \mu \sin [\lambda d(j-k)] \mathrm{e}^{-2 v f} \mathrm{~d} \mu, \\
S_{m n}^{j k} & =2(-1)^{m+n} f_{0}^{\infty} \frac{v+K}{v-K} \sinh m \mu \sinh n \mu \cos [\lambda d(j-k)] \mathrm{e}^{-2 v f} \mathrm{~d} \mu .
\end{aligned}
$$

Here $\epsilon_{0}=1, \epsilon_{n}=2$ for $n \geqslant 1$. For computational purposes we note that

$$
\begin{aligned}
P_{m n}^{j k}= & \epsilon_{m}(-1)^{m+n}\left[\int _ { 0 } ^ { b } \left((\nu+K) \lambda^{-1} \cosh m \mu \cosh n \mu \cos [\lambda d(j-k)] \mathrm{e}^{-2 v f}\right.\right. \\
& \left.-2(K / \alpha) \cosh m \gamma \cosh n \gamma \cos [\alpha d(j-k)] \mathrm{e}^{-2 K f}\right) \frac{\lambda \mathrm{d} \mu}{v-K} \\
& +\int_{b}^{\infty} \frac{v+K}{v-K} \cosh m \mu \cosh n \mu \cos [\lambda d(j-k)] \mathrm{e}^{-2 v f} \mathrm{~d} \mu \\
& \left.+2 \pi \mathrm{i}(K / \alpha) \cosh m \gamma \cosh n \gamma \cos [\alpha d(j-k)] \mathrm{e}^{-2 K f}\right]
\end{aligned}
$$

where $K=\ell \cosh \gamma$ and $\cosh b=(2 K / \ell)-1$. Similar expressions can be derived for $Q_{m n}^{j k}, R_{m n}^{j k}$ and $S_{m n}^{j k}$.

To re-expand the singular terms about $x=k d, z=-f$ we use Graf's addition theorem which here takes the form

$$
\begin{aligned}
\mathrm{K}_{n}\left(\ell r_{j}\right) \exp \left(\mathrm{i} n \theta_{j}\right)=\sum_{m=-\infty}^{\infty} \mathrm{i}^{m+n} \mathrm{~K}_{n-m}(\mid j & -k \mid \ell d) \exp \left[\mathrm{i}(n-m) \chi_{j k}\right] \\
& \times \mathrm{I}_{m}\left(\ell r_{k}\right) \exp \left(\mathrm{i} m \theta_{k}\right), \quad j \neq k
\end{aligned}
$$

We can thus show that, for $j \neq k$ and $r_{k}<\min (2 f,|j-k| d)$,

$$
\begin{aligned}
& \phi_{n}^{j}=\sum_{m=0}^{\infty}\left(P_{m n}^{j k}+D_{m n}^{j k}\right) \mathrm{I}_{m}\left(\ell r_{k}\right) \cos m \theta_{k}+\sum_{m=1}^{\infty}\left(E_{m n}^{j k}-Q_{m n}^{j k}\right) \mathrm{I}_{m}\left(\ell r_{k}\right) \sin m \theta_{k}, \\
& \psi_{n}^{j}=\sum_{m=0}^{\infty}\left(R_{m n}^{j k}+F_{m n}^{j k}\right) \mathrm{I}_{m}\left(\ell r_{k}\right) \cos m \theta_{k}+\sum_{m=1}^{\infty}\left(S_{m n}^{j k}+G_{m n}^{j k}\right) \mathrm{I}_{m}\left(\ell r_{k}\right) \sin m \theta_{k}
\end{aligned}
$$


where, with $K_{n}^{j k}=\mathrm{K}_{n}(|j-k| \ell d)$,

$$
\begin{aligned}
D_{m n}^{j k} & =\frac{\epsilon_{m}}{2}\left(\cos \left[(n+m) \frac{\pi}{2}\right] K_{n-m}^{j k}+\cos \left[(n-m) \frac{\pi}{2}\right] K_{n+m}^{j k}\right), \\
E_{m n}^{j k} & =\operatorname{sgn}(k-j)\left(-\sin \left[(n+m) \frac{\pi}{2}\right] K_{n-m}^{j k}+\sin \left[(n-m) \frac{\pi}{2}\right] K_{n+m}^{j k}\right), \\
F_{m n}^{j k} & =\frac{\epsilon_{m}}{2} \operatorname{sgn}(k-j)\left(\sin \left[(n+m) \frac{\pi}{2}\right] K_{n-m}^{j k}+\sin \left[(n-m) \frac{\pi}{2}\right] K_{n+m}^{j k}\right), \\
G_{m n}^{j k} & =\cos \left[(n+m) \frac{\pi}{2}\right] K_{n-m}^{j k}-\cos \left[(n-m) \frac{\pi}{2}\right] K_{n+m}^{j k} .
\end{aligned}
$$

Note that $D_{m n}^{j k}=G_{m n}^{j k}=0$ if $m+n$ is odd, while $E_{m n}^{j k}=F_{m n}^{j k}=0$ if $m+n$ is even.

\subsection{Infinite array}

We seek a solution in the form

$$
\phi=\sum_{j=-\infty}^{\infty} \mathrm{e}^{\mathrm{i} \beta j d}\left(\sum_{n=0}^{\infty} \tilde{\xi}_{n} \phi_{n}^{j}+\sum_{n=1}^{\infty} \tilde{\eta}_{n} \psi_{n}^{j}\right),
$$

with $\tilde{\xi}_{n}$ and $\tilde{\eta}_{n}$ to be determined and $0 \leqslant \beta d \leqslant \pi$ as before. Incorporating (4.5) and using the Poisson summation formula shows that, for $z>-f$, provided $v_{j} \neq K$ for any $j$,

$$
\phi=\sum_{j=-\infty}^{\infty} \mathscr{A}_{j} \frac{v_{j} \cosh v_{j} z+K \sinh v_{j} z}{v_{j}\left(v_{j}-K\right)} \exp \left(\mathrm{i} \beta_{j} x-v_{j} f\right)
$$

where

$$
\mathscr{A}_{j}=\sum_{n=0}^{\infty} \frac{2 \pi}{d}(-1)^{n}\left(\tilde{\xi}_{n} \cosh n \mu_{j}+\mathrm{i} \tilde{\eta}_{n} \sinh n \mu_{j}\right)
$$

Here

$$
\mu_{j}=\sinh ^{-1}\left(\beta_{j} / \ell\right)=\ln \left[\frac{\beta_{j}}{\ell}+\left(\frac{\beta_{j}^{2}}{\ell^{2}}+1\right)^{1 / 2}\right],
$$

and we have written

$$
v_{j}=\ell \cosh \mu_{j}=\left(\ell^{2}+\beta_{j}^{2}\right)^{1 / 2} .
$$

The apparent singularities at $v_{j}=K$ are removable, since it can be shown that if $v_{j}=K$ for a particular $j$, then $\mathscr{A}_{j}=0$.

As in the $\ell=0$ case, we need to satisfy only the boundary condition on one cylinder (which we take to be the one at the origin), and then all the others are satisfied because of quasi-periodicity. We define

$$
\hat{P}_{m n}=\sum_{j=-\infty}^{\infty} \mathrm{e}^{\mathrm{i} \beta j d} P_{m n}^{j 0},
$$

and we also similarly define $\hat{Q}_{m n}, \hat{R}_{m n}$ and $\hat{S}_{m n}$. Further, we define

$$
\hat{D}_{m n}=\sum_{j=-\infty}^{\infty} \mathrm{e}^{\mathrm{i} \beta j d} D_{m n}^{j 0}
$$


and we similarly define $\hat{E}_{m n}, \hat{F}_{m n}$ and $\hat{G}_{m n}$. Setting $\partial \phi / \partial r=0$ on $r=a$ then yields

$$
\begin{aligned}
& \xi_{m}+\sum_{n=0}^{\infty} Z_{m n} \xi_{n}\left(\hat{P}_{m n}+\hat{D}_{m n}\right)+\sum_{n=1}^{\infty} Z_{m n} \eta_{n}\left(\hat{R}_{m n}+\hat{F}_{m n}\right)=0, \quad m \geqslant 0, \\
& \eta_{m}+\sum_{n=0}^{\infty} Z_{m n} \xi_{n}\left(\hat{E}_{m n}-\hat{Q}_{m n}\right)+\sum_{n=1}^{\infty} Z_{m n} \eta_{n}\left(\hat{S}_{m n}+\hat{G}_{m n}\right)=0, \quad m \geqslant 1,
\end{aligned}
$$

where we have written $\xi_{m}=\tilde{\xi}_{m} \mathrm{~K}_{m}^{\prime}(\ell a), \eta_{m}=\tilde{\eta}_{m} \mathrm{~K}_{m}^{\prime}(\ell a)$ and $Z_{m n}=\mathrm{I}_{m}^{\prime}(\ell a) / \mathrm{K}_{n}^{\prime}(\ell a)$. Truncated versions of these equations will be used in $\S 5$ to determine numerical approximations to $\xi_{m}$ and $\eta_{m}$.

It is helpful to define

$$
\hat{K}_{n}= \begin{cases}2 \sum_{j=1}^{\infty} \mathrm{K}_{n}(j \ell d) \cos \beta j d, & n \text { even } \\ -2 \mathrm{i} \sum_{j=1}^{\infty} \mathrm{K}_{n}(j \ell d) \sin \beta j d, & n \text { odd }\end{cases}
$$

If $n+m$ is odd, then $\hat{D}_{m n}=\hat{G}_{m n}=0$. On the other hand, if $n+m$ is even we have

$$
\begin{aligned}
& \hat{D}_{m n}=\frac{\epsilon_{m}}{2}\left[(-1)^{(n+m) / 2} \hat{K}_{n-m}+(-1)^{(n-m) / 2} \hat{K}_{n+m}\right], \\
& \hat{G}_{m n}=(-1)^{(n+m) / 2} \hat{K}_{n-m}-(-1)^{(n-m) / 2} \hat{K}_{n+m},
\end{aligned}
$$

and these are clearly real. Similarly if $n+m$ is even, then $\hat{E}_{m n}=\hat{F}_{m n}=0$, whereas if $n+m$ is odd we have

$$
\begin{aligned}
& \hat{E}_{m n}=-(-1)^{(n+m-1) / 2} \hat{K}_{n-m}+(-1)^{(n-m-1) / 2} \hat{K}_{n+m}, \\
& \hat{F}_{m n}=\frac{\epsilon_{m}}{2}\left[(-1)^{(n+m-1) / 2} \hat{K}_{n-m}+(-1)^{(n-m-1) / 2} \hat{K}_{n+m}\right],
\end{aligned}
$$

which are imaginary. Application of the Poisson summation formula shows that

$$
\begin{aligned}
& \hat{P}_{m n}=\epsilon_{m} \pi(-1)^{m+n} \sum_{j=-\infty}^{\infty} \frac{v_{j}+K}{v_{j}-K} \cosh m \mu_{j} \cosh n \mu_{j} \frac{\mathrm{e}^{-2 v_{j} f}}{v_{j} d} \\
& \hat{Q}_{m n}=\frac{2}{\epsilon_{n}} \hat{R}_{n m}=2 \pi \mathrm{i}(-1)^{m+n} \sum_{j=-\infty}^{\infty} \frac{v_{j}+K}{v_{j}-K} \sinh m \mu_{j} \cosh n \mu_{j} \frac{\mathrm{e}^{-2 v_{j} f}}{v_{j} d}, \\
& \hat{S}_{m n}=2 \pi(-1)^{m+n} \sum_{j=-\infty}^{\infty} \frac{v_{j}+K}{v_{j}-K} \sinh m \mu_{j} \sinh n \mu_{j} \frac{\mathrm{e}^{-2 v_{j} f}}{v_{j} d} .
\end{aligned}
$$

All the quantities in (4.26) and (4.27) are then given in terms of exponentially convergent series.

To truncate (4.26) and (4.27) to a finite linear system we write

$$
\left(x_{1}, x_{2}, \ldots, x_{2 N}\right)=\left(\xi_{0}, \mathrm{i} \eta_{1}, \xi_{1}, \mathrm{i} \eta_{2}, \ldots, \xi_{N-1}, \mathrm{i} \eta_{N}\right) .
$$


In other words $\xi_{m}=x_{2 m+1}$ and $\eta_{m}=-\mathrm{i} x_{2 m}$. Then (4.26) and (4.27) become

$$
\begin{array}{r}
x_{2 m+1}+\sum_{n=0}^{N-1} Z_{m n} x_{2 n+1}\left(\hat{P}_{m n}+\hat{D}_{m n}\right)-\mathrm{i} \sum_{n=1}^{N} Z_{m n} x_{2 n}\left(\hat{R}_{m n}+\hat{F}_{m n}\right)=0, \\
0 \leqslant m \leqslant N-1, \\
x_{2 m}+\mathrm{i} \sum_{n=0}^{N-1} Z_{m n} x_{2 n+1}\left(\hat{E}_{m n}-\hat{Q}_{m n}\right)+\sum_{n=1}^{N} Z_{m n} x_{2 n}\left(\hat{S}_{m n}+\hat{G}_{m n}\right)=0, \\
1 \leqslant m \leqslant N .
\end{array}
$$

Thus we require the determinant of the $2 N \times 2 N$ matrix $\boldsymbol{M}=\left(M_{i j}\right)_{i, j=1}^{2 N}$ to vanish, where

$$
\left.\begin{array}{rl}
M_{2 m-1,2 n-1} & =\delta_{m n}+Z_{m-1, n-1}\left(\hat{P}_{m-1, n-1}+\hat{D}_{m-1, n-1}\right), \\
M_{2 m-1,2 n} & =-\mathrm{i} Z_{m-1, n}\left(\hat{R}_{m-1, n}+\hat{F}_{m-1, n}\right), \\
M_{2 m, 2 n-1} & =\mathrm{i} Z_{m, n-1}\left(\hat{E}_{m, n-1}-\hat{Q}_{m, n-1}\right), \\
M_{2 m, 2 n} & =\delta_{m n}+Z_{m n}\left(\hat{S}_{m n}+\hat{G}_{m n}\right),
\end{array}\right\}
$$

in which all the entries are real. It can be shown that as $\ell \rightarrow 0$ the system for oblique waves reduces to that for $\ell=0$.

\subsection{Finite array}

We seek a solution in the form

$$
\phi=\phi_{i n c}+\phi_{s c}=\phi_{i n c}+\sum_{j=0}^{M-1}\left(\sum_{n=0}^{\infty} \tilde{\xi}_{n}^{j} \phi_{n}^{j}+\sum_{n=1}^{\infty} \tilde{\eta}_{n}^{j} \psi_{n}^{j}\right),
$$

in which (see Linton \& McIver 2001, (3.21))

$$
\phi_{i n c}=\mathrm{e}^{\mathrm{i} \alpha x} \mathrm{e}^{K z}=\mathrm{e}^{\mathrm{i} k \alpha d} \mathrm{e}^{-K f} \sum_{n=0}^{\infty} \epsilon_{n}(-1)^{n} \mathrm{I}_{n}\left(\ell r_{k}\right) \cos n\left(\theta_{k}+\mathrm{i} \gamma\right) .
$$

Setting $\partial \phi / \partial r_{k}=0$ on $r=a_{k}$ then yields

$$
\begin{aligned}
\xi_{m}^{k}+ & \sum_{j=0}^{M-1}\left(\sum_{n=0}^{\infty} Z_{m n} \xi_{n}^{j}\left(P_{m n}^{j k}+D_{m n}^{j k}\right)+\sum_{n=1}^{\infty} Z_{m n} \eta_{n}^{j}\left(R_{m n}^{j k}+F_{m n}^{j k}\right)\right) \\
& =-\epsilon_{m}(-1)^{m} \mathrm{e}^{\mathrm{i} k \alpha d} \mathrm{e}^{-K f} \mathrm{I}_{m}^{\prime}(\ell a) \cosh m \gamma, \quad m \geqslant 0, k=0, \ldots, M-1, \\
\eta_{m}^{k}+ & \sum_{j=0}^{M-1}\left(\sum_{n=0}^{\infty} Z_{m n} \xi_{n}^{j}\left(E_{m n}^{j k}-Q_{m n}^{j k}\right)+\sum_{n=1}^{\infty} Z_{m n} \eta_{n}^{j}\left(S_{m n}^{j k}+G_{m n}^{j k}\right)\right) \\
= & 2 \mathrm{i}(-1)^{m} \mathrm{e}^{\mathrm{i} k \alpha d} \mathrm{e}^{-K f} \mathrm{I}_{m}^{\prime}(\ell a) \sinh m \gamma, \quad m \geqslant 1, k=0, \ldots, M-1,
\end{aligned}
$$

where we have defined $D_{m n}^{k k}=E_{m n}^{k k}=F_{m n}^{k k}=G_{m n}^{k k}=0$. This can readily be truncated to a $2 M N \times 2 M N$ system by restricting $m$ and $n$ in (4.42) and (4.43) to range between 0 and $N-1$ or 1 and $N$ as appropriate. 
From (4.40) and Linton \& McIver (2001, (B.112) and (B.118)), we have that as $x \rightarrow \pm \infty$,

$$
\begin{aligned}
\phi_{s c} \sim \frac{2 \pi K}{\alpha} \exp [K(z- & f)] \sum_{j=0}^{M-1} \exp [ \pm \mathrm{i} \alpha(x-j d)] \\
& \times\left(\sum_{n=0}^{\infty} \mathrm{i}(-1)^{n} \tilde{\xi}_{n}^{j} \cosh n \gamma \mp \sum_{n=1}^{\infty}(-1)^{n} \tilde{\eta}_{n}^{j} \sinh n \gamma\right) .
\end{aligned}
$$

Hence the reflection and transmission coefficients are

$$
\begin{aligned}
& R_{M}=\frac{2 \pi K}{\alpha} \mathrm{e}^{-K f} \sum_{j=0}^{M-1} \mathrm{e}^{\mathrm{i} j \alpha d}\left(\sum_{n=0}^{\infty} \mathrm{i}(-1)^{n} \tilde{\xi}_{n}^{j} \cosh n \gamma+\sum_{n=1}^{\infty}(-1)^{n} \tilde{\eta}_{n}^{j} \sinh n \gamma\right), \\
& T_{M}=1+\frac{2 \pi K}{\alpha} \mathrm{e}^{-K f} \sum_{j=0}^{M-1} \mathrm{e}^{-\mathrm{i} j \alpha d}\left(\sum_{n=0}^{\infty} \mathrm{i}(-1)^{n} \tilde{\xi}_{n}^{j} \cosh n \gamma-\sum_{n=1}^{\infty}(-1)^{n} \tilde{\eta}_{n}^{j} \sinh n \gamma\right) .
\end{aligned}
$$

As in $\S 3$, alternative expressions for the reflection and transmission coefficients can be obtained in terms of the horizontal and vertical exciting forces on the cylinders. In fact (3.35) remains true in this case if $K d$ is replaced by $\alpha d$ in the expression for $T_{M}$.

\section{Numerical results}

The size of the systems of equations derived in $\S \S 3$ and 4 involve the truncation parameter $N$. In all the results presented below we have used $N=8$. This means that 16 multipoles are used to represent the scattered field generated by each cylinder. For all the parameter values that we consider, which does not include the cases of high frequencies or cylinders very close to the free surface or to each other, this is more than sufficient to guarantee high accuracy. As an example, corresponding to $K a=0.6$ on the $M=10$ curve in figure 1, truncation parameters of $N=2,4,8$ and 16 give values of the reflected energy as, respectively, $|R|^{2} \approx 0.21642,0.17257,0.17251$ and 0.17251 . Results are presented for up to 60 cylinders, a case which thus requires the solution to a $960 \times 960$ system of equations. Reflection and transmission coefficients were always checked by calculating them both from the series expressions and in terms of the exciting forces, and energy conservation was always satisfied to a great degree of accuracy.

We begin with the $\ell=0$ case. The transfer matrix analysis in $\S 2$ (based on a widespacing approximation) suggests that Bragg-type resonance is unlikely for submerged cylinders in deep water because, as is well known, $|R|=0$ for a single submerged cylinder for any submergence depth and any frequency (Dean 1948). That resonant reflection does occur is clear from figure 1 which shows the modulus of the reflection coefficient as a function of $K a$ for 5,10 and 20 cylinders when $f / d=0.375$ and $a / d=0.25$. A wavelength of twice the geometrical period would correspond to $K a=\pi / 4$ in this case, whereas the figure shows that the first resonance actually occurs for $K a \approx 0.57$, which corresponds to a wavelength of roughly 2.75 times the geometrical period. This shift is consistent with (2.16) if we interpret this condition as suggesting that resonance is most likely to occur when $|\cos \chi|=1$. When $K a=0.57$ we can compute the phase of the transmission coefficient as approximately 0.93 , which leads to a value of $\chi$ which is just greater than $\pi$. A similar calculation can be performed for the higher resonances, which according to Bragg's law correspond 


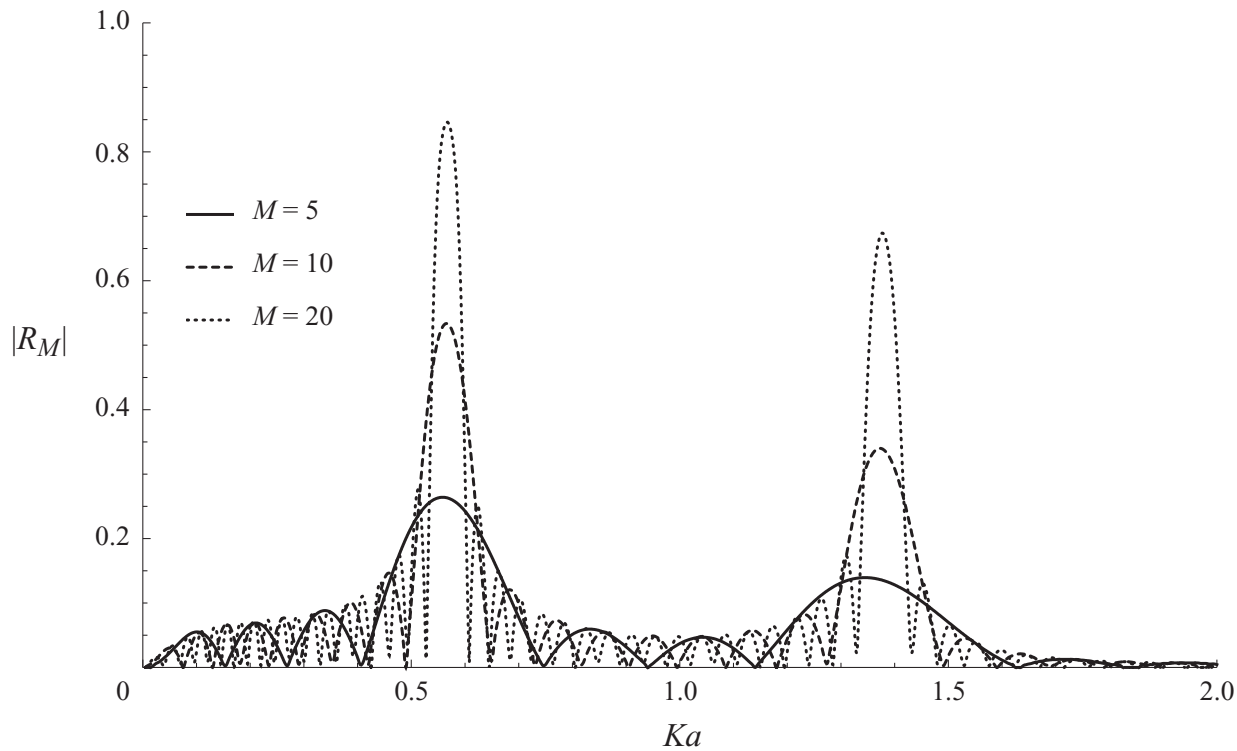

FIGURE 1. Modulus of reflection coefficient as a function of $K a$ for $f / d=0.375, a / d=0.25$.

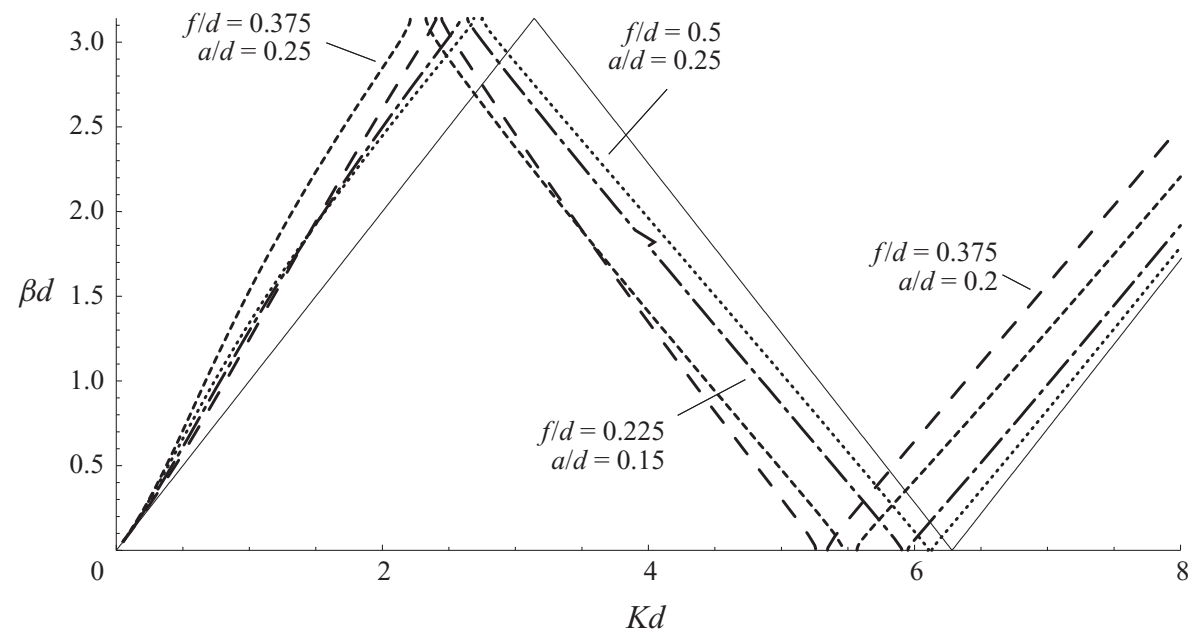

FIGURE 2. Dispersion curves showing $\beta d$ as a function of $K d$. The thin solid lines are $\beta d=K d$, $\beta d=2 \pi-K d$ and $\beta d=K d-2 \pi$. The dotted lines are for $f / d=0.5, a / d=0.25$; the shorter dashed lines are for $f / d=0.375, a / d=0.25$; the longer dashed lines are for $f / d=0.375$, $a / d=0.2$; the dash-dotted lines are for $f / d=0.225, a / d=0.15$.

to $\lambda=2 d / n, n=2,3, \ldots$. From figure 1 the second resonance occurs at $K a \approx 1.38$ $(K d \approx 5.52)$ which is consistent with $\chi=\arg T+K d=2 \pi$, since $\arg T \approx 0.72$ when $K a=1.38$.

To see whether it is possible to have $\left|T_{M}\right| \rightarrow 0$ as $M \rightarrow \infty$ we compute dispersion curves based on solving (3.25) and (3.26). We fix $K d$ and search for values of $\beta d \in[0, \pi]$ for which a non-trivial solution to this coupled system exists. Results for four pairs of values of $f / d$ and $a / d$ are shown in figures 2 and 3 and in table 1 . Figure 2 shows dispersion curves over the range $0<K d<8$, together with the zigzag 


$\begin{array}{llll}f / d & a / d & \text { Gap } 1(K d) & \text { Gap 2 }(K d) \\ 0.5 & 0.25 & (2.687,2.794) & (6.105,6.130) \\ 0.375 & 0.25 & (2.210,2.328) & (5.457,5.569) \\ 0.375 & 0.2 & (2.587,2.640) & (5.904,5.951) \\ 0.225 & 0.15 & (2.402,2.446) & (5.257,5.347)\end{array}$

TABLE 1. Computed values for the band gaps shown in figures 2 and 3.
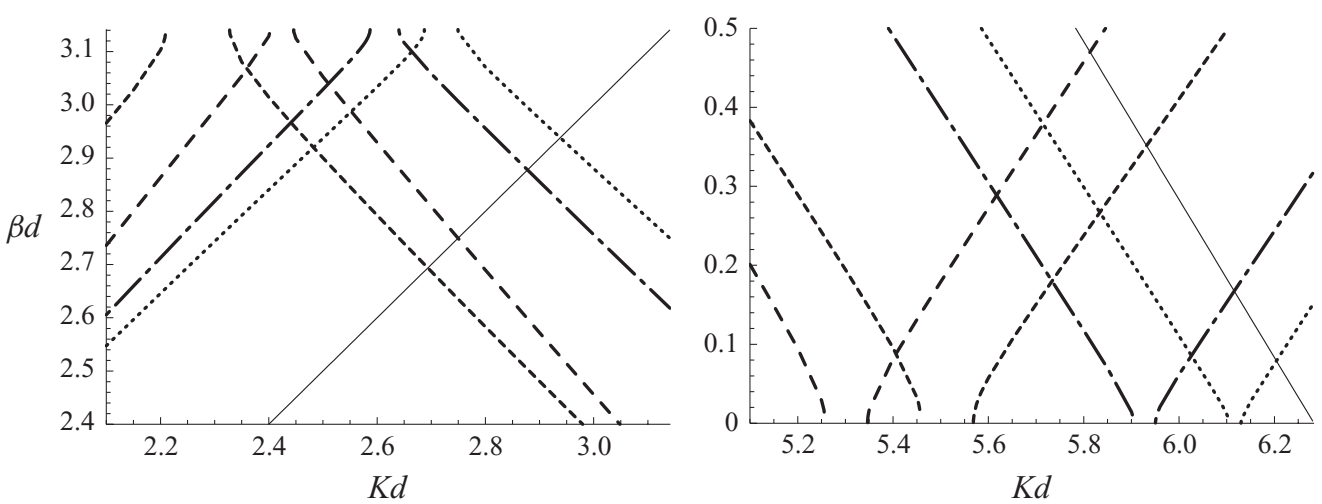

FIGURE 3. Close-up of the first two band gaps.

line made up by the curves $\beta d=K d, \beta d=2 \pi-K d\left(\beta_{-1}=-K\right)$ and $\beta d=K d-2 \pi$ $\left(\beta_{1}=K\right)$, which all the other curves essentially follow. The first 'point' on this zigzag line is at $K d=\pi$ which corresponds to a wavelength of exactly twice the geometrical period. All the curves shown exhibit band gaps. In other words there are ranges of values of $K d$ for which there are no allowable values of $\beta d$, and these intervals are shown in close-up in figure 3 and collected together in table 1 . If we consider the case $f / d=0.375, a / d=0.25$ (which corresponds to the parameters in figure 1 ), then we see that the resonances in figure 1 occur at values of $K d(\approx 2.28$ and 5.5$)$ in the middle of the band gaps.

The existence of these band gaps means that we would expect $\left|T_{M}\right| \rightarrow 0$ as $M \rightarrow \infty$ whenever $K d$ lies in one of the gaps, and this is indeed the case, as shown in figure 4, which in fact shows the transmitted energy $\left|T_{M}\right|^{2}$ as a function of $M$ for a range of values of $K d$ (and with $f / d=0.375$ and $a / d=0.25$ as in figure 1). Three of these $K d$ values lie within the band gap (see table 1), and for these the energy decays monotonically as $M$ increases. For all the other values of $K d$ the transmitted energy oscillates between 1 and some finite value, that value (and the frequency of the oscillations) decreasing as the edge of the band gap is approached.

Next we turn to the case $\ell>0$. Figure 5 shows dispersion curves $\beta d$ as a function of $K d$ for various values of $\ell d$ based on solving (4.37) and (4.38). The most obvious difference between this and the case $\ell=0$ (figure 2) is that as $\ell$ increases from zero a gap appears at the origin. Thus, for a given $\ell>0$ there is a minimum value of the frequency for which modes are possible. However, the minimum value of $K d$ is less than $\ell d$ in all cases that we have computed, and therefore it is not possible to consider a scattering problem corresponding to a frequency in this gap. Computed values for the band gaps shown in figure 5 are shown in table 2 . For the second and third band gaps we also show the intervals of $K d$ in which the approximate condition 


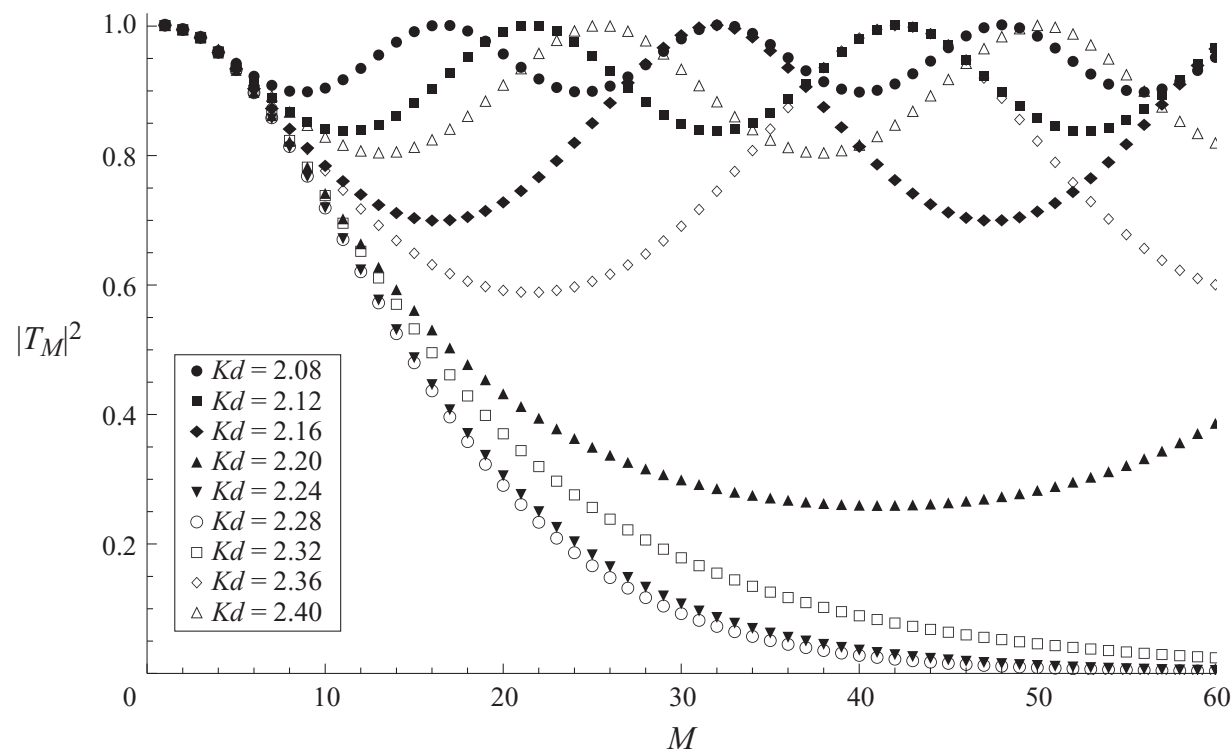

FIGURE 4. Transmitted energy as a function of the number of cylinders in the array for $f / d=0.375, a / d=0.25$.

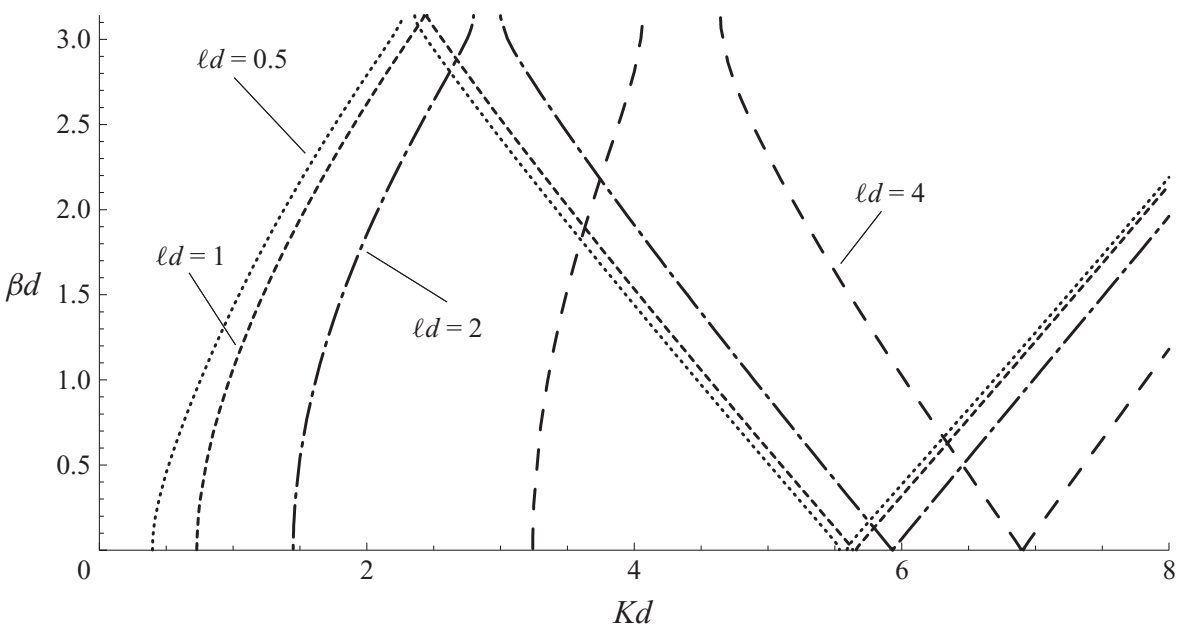

FIGURE 5. Dispersion curves showing $\beta d$ as a function of $K d$. The dotted lines are for $\ell d=0.5$; the shorter dashed lines are for $\ell d=1$; the dash-dotted lines are for $\ell d=2$; the longer dashed lines are for $\ell d=4$. In all cases $f / d=0.375, a / d=0.25$.

for stop bands (2.16) is satisfied. This can only be done when $K>\ell$, which is why no approximate values appear for the first band gap. The transfer matrix analysis clearly provides an excellent guide as to the location of the band gaps.

The relation between these band gaps and scattering problems for oblique waves in which $\left|T_{M}\right| \rightarrow 0$ as $M \rightarrow \infty$ is less clear than in the case $\ell=0$. This is because in such a scattering problem one would usually treat either the incident wave angle or the frequency as fixed and then vary the other of these two parameters, in which case $\ell=K \sin \varphi$ is not constant. Nevertheless, the association can still be made. Figure 6 shows the transmitted energy $\left|T_{M}\right|^{2}$ as a function of $M$ for a range of values of 


$\begin{array}{lccc}\ell d & \text { Gap 1 }(K d) & \text { Gap 2 }(K d) & \text { Gap 3 }(K d) \\ 0.5 & (0,0.396) & (2.266,2.358) & (5.535,5.592) \\ & & (2.262,2.270) & (5.592,5.595) \\ 1 & (0,0.728) & (2.429,2.448) & (5.635,5.661) \\ 2 & (0,1.450) & (2.377,2.431) & (5.661,5.671) \\ & & (2.798,3.000) & (5.930,5.931) \\ 4 & (0,3.240) & (2.765,3.001) & (5.931,5.943) \\ & & (4.061,4.644) & (6.893,6.911) \\ & & (4.057,4.645) & (6.896,6.910)\end{array}$

TABLE 2. Computed values for the band gaps shown in figure 5. Where there are two intervals listed, the lower values are computed from the approximate condition (2.16).

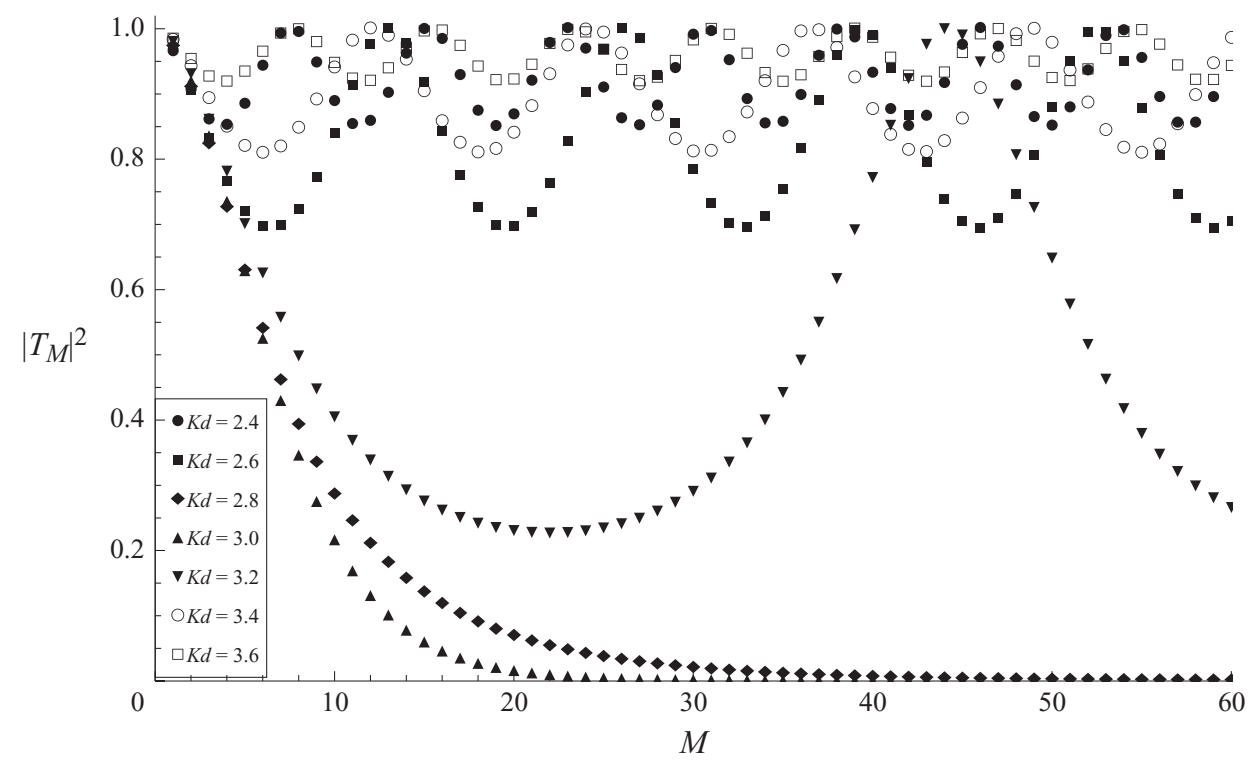

FIGURE 6. Transmitted energy as a function of the number of cylinders in the array for $f / d=0.375, a / d=0.25$ and $\varphi=45^{\circ}$.

$K d$ when $f / d=0.375$ and $a / d=0.25$ and when the incident wave angle $\varphi$ is fixed at $45^{\circ}$. Two of the values of $K d$ used in the figure appear to lead to a decaying transmission coefficient, namely $K d=2.8$ and $K d=3$. For the former of these we have $\ell d=2.8 / \sqrt{2} \approx 1.98$, and if we compute the band gap for $\ell d=1.98$ we find that it is $(2.783,2.991)$, which includes $K d=2.8$, whereas for the latter we have $\ell d=3 / \sqrt{2} \approx 2.12$, and if we compute the band gap for $\ell d=2.12$ we find that it is (2.854, 3.084), which includes $K d=3$.

We can also plot dispersion curves showing $\beta d$ as a function of $\ell d$ for various values of $K d$. From the general structure of the dispersion curves shown in figure 5 we can expect that there will be a different structure to the $\beta d-\ell d$ curves, depending on the size of $K d$, with more band gaps appearing as $K d$ increases. This is borne out by the curves shown in figure 7 , which is for the case $f / d=0.5, a / d=0.25$. When $K d=2$ there are no band gaps; for $K d=3,4,5$ and 6 there is one band gap, while for $K d=7$ there are two. 


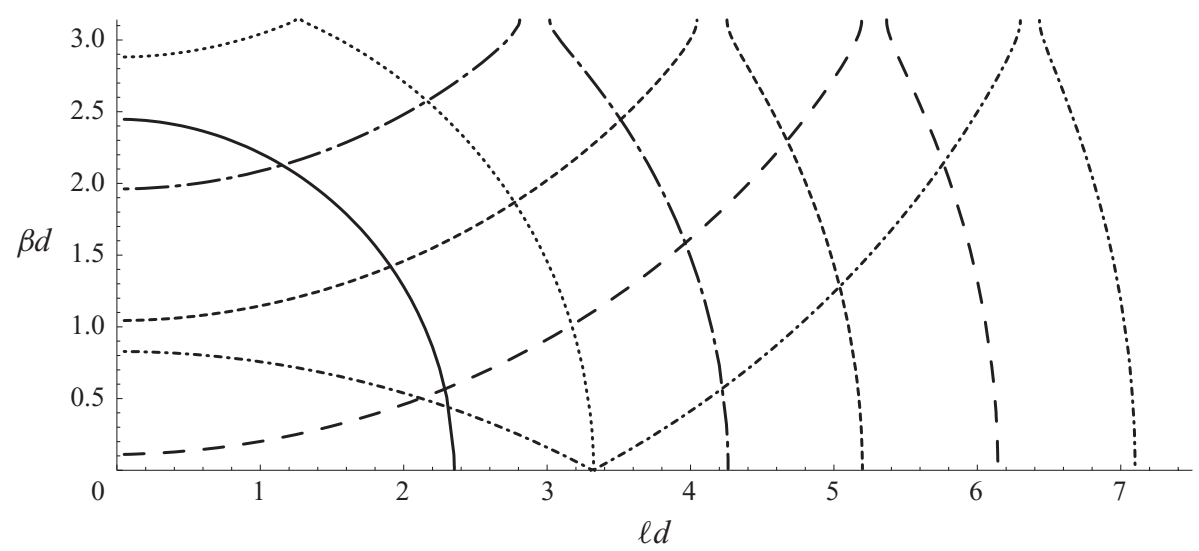

FIGURE 7. Dispersion curves showing $\beta d$ as a function of $\ell d$ for various values of $K d$ when $f / d=0.5, a / d=0.25$. The solid line is for $K d=2$, the dotted line is for $K d=3$, and continuing to the right we have curves for $K d=4,5,6$ and 7 .

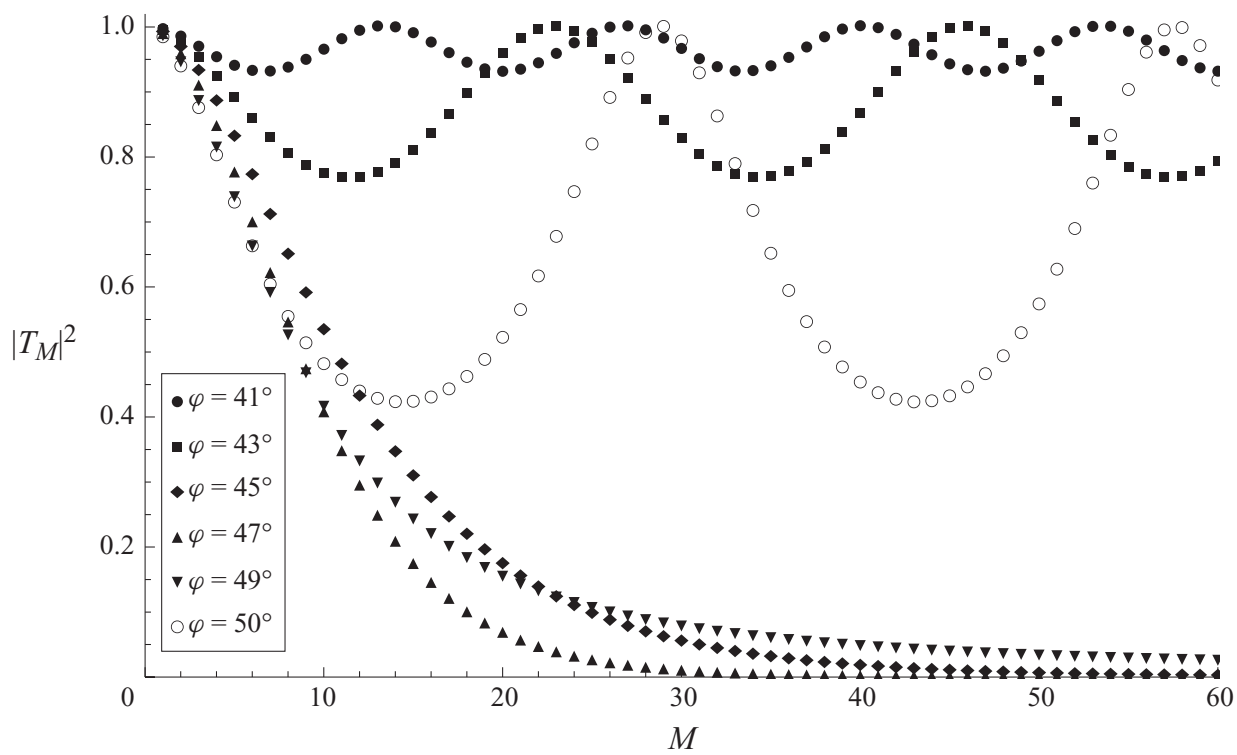

FIGURE 8. Transmitted energy as a function of the number of cylinders in the array for $K a=1, f / d=0.5, a / d=0.25$.

The curves in figure 7 allow us to determine the direction in which the energy associated with these modes travels. If we write $\boldsymbol{k}=(\beta, \ell)$ for the wavenumber vector, then the group velocity for any mode is simply $\mathrm{d} \omega / \mathrm{d} \boldsymbol{k}$, and since the curves in figure 7 are constant-frequency contours, the group velocity is always perpendicular to these curves, in the direction of increasing $K d$. All the curves hit the $\ell d=0$ axis at right angles, as they must because then there is no $y$-variation and the modes propagate along the $x$-axis. (The figure shows only half the picture; one needs to reflect about either $\beta d=0$ or $\beta d=\pi$ to see all the possible modes.) When $K d=2$ the direction of the modes gradually shifts round until the mode cuts off, at which point the propagation is entirely in the $y$-direction. For higher values of $K d$ we can have fundamentally different modes which have the same frequency and which propagate 
in the same direction. Thus, for example, when $K d=4$ there are three modes for which the energy propagates in the $y$-direction, two for which $\beta d=\pi$ (on either side of the band gap) and one for which $\beta d=0$.

In figure 7, the band gap for $K d=4$ corresponds to $\ell d \in(2.808,3.017)$, which is equivalent to $\varphi \in\left(44.6^{\circ}, 49.0^{\circ}\right)$. Thus if we fix the frequency at $K d=4$ and vary the incident wave angle we would expect $\left|T_{M}\right| \rightarrow 0$ as $M \rightarrow \infty$ if $\varphi$ lies in this interval, but not otherwise. That this is the case is shown in figure 8 .

\section{Conclusion}

The existence of separate pass and stop bands associated with wave propagation through periodic media is a common phenomenon, though it appears to have received rather little attention in the context of the full linear theory of water waves. In this paper we have focused on water waves propagating over periodic arrays of submerged horizontal circular cylinders in deep water, considering waves travelling at an oblique angle as well as normal to the cylinder axes.

The fact that submerged circular cylinders are weak reflectors makes this configuration particularly interesting. In a sense they are the weakest of all reflectors, since the reflection coefficient for a single cylinder (with normal incidence) is zero for all frequencies. Thus it is not obvious that there will be any stop bands. The fact that these are there, as we have shown, indicates that the evanescent interactions between the cylinders must play a crucial role in their creation, and heuristic arguments that are often put forward to explain Bragg resonance are inadequate in this case. A zero reflection coefficient does not imply that the waves are unaffected by the cylinder, and it is well known that there is a phase shift as the wave propagates over such a structure. The existence of this phase shift means that the standard Bragg law, which can be thought of as a first approximation to the location of a band gap, needs to be modified.

The multipole expansion technique has been used to solve both the case of an infinite array and that of a finite array of cylinders. This approach appears to work particularly well for the infinite-array case and normal incidence, since some of the infinite sums that are required can be evaluated in closed form. The infinite linear systems that are obtained must be truncated in order to compute the numerical results, but in principle, we can determine the solution to the full linear problem with any desired accuracy. Numerical results show that band gaps do exist for both normally propagating and oblique waves, and we have shown how this corresponds to cases in which the energy which is transmitted across a finite array tends to zero as the size of the array increases.

Apart from examining the behaviour of $\left|T_{M}\right|$ as $M \rightarrow \infty$ we have not considered the problem of a semi-infinite array of cylinders in any detail. This could be done using the methods described in Porter \& Evans (2006) and would be an interesting extension to the current analysis.

Thanks are due to Paul Martin for bringing the work of Schnute (1967) to the attention of the author.

\section{REFERENCES}

Abramowitz, M. \& Stegun, I. A. 1965 Handbook of Mathematical Functions. Dover.

AN, Z. \& YE, Z. 2004 Band gaps and localization of water waves over one-dimensional topographical bottoms. Appl. Phys. Lett. 84 (15), 2952-2954. 
Bennetts, L. G., Biggs, N. R. T. \& Porter, D. 2009 The interaction of flexural-gravity waves with periodic geometries. Wave Motion 46 (1), 57-73.

Chamberlain, P. G. \& Porter, D. 1995 Decomposition methods for wave scattering by topography with application to ripple beds. Wave Motion 22, 201-214.

Chen, L.-S., Kuo, C.-H., Ye, Z. \& Sun, X. 2004 Band gaps in the propagation and scattering of surface water waves over cylindrical steps. Phys. Rev. E 69, 066308.

CHou, T. 1998 Band structure of surface flexural-gravity waves along periodic interfaces. J. Fluid Mech. 369, 333-350.

Davies, A. G. 1982 The reflection of wave energy by undulations on the seabed. Dyn. Atmos. Oceans 6, 207-232.

Davies, A. G. \& Heathershaw, A. D. 1984 Surface-wave propagation over sinusoidally varying topography. J. Fluid Mech. 144, 419-443.

DeAn, W. R. 1948 On the reflexion of surface waves by a submerged circular cylinder. Proc. Camb. Phil. Soc. 44, 483-491.

Devillard, P., Dunlop, F. \& Souillard, B. 1988 Localization of gravity waves on a channel with random bottom. J. Fluid Mech. 186, 521-538.

Evans, D. V. 1990 The wide spacing approximation applied to multiple scattering and sloshing problems. J. Fluid Mech. 210, 647-658.

Garnaud, X. \& MeI, C. C. 2010 Bragg scattering and wave-power extraction by an array of small buoys. Proc. R. Soc. Lond. A 466 (2113), 79-106.

Hunter, G. K. 2004 Light is a Messenger: The Life and Science of William Lawrence Bragg. Oxford University Press.

Levine, H. 1965 Scattering of surface waves by a submerged circular cylinder. J. Math. Phys. 6 (8), 1231-1243.

Linton, C. M. \& MCIVER, P. 2001 Handbook of Mathematical Techniques for Wave/Structure Interactions. Chapman \& Hall/CRC.

Markoš, P. \& Soukoulis, C. M. 2008 Wave Propagation: From Electrons to Photonic Crystals and Left-Handed Materials. Princeton University Press.

MCIvER, P. 1990 The scattering of long water waves by a group of submerged, horizontal cylinders. Q. J. Mech. Appl. Math. 43 (4), 499-515.

MCIvER, P. 2000 Water-wave propagation through an infinite array of cylindrical structures. J. Fluid Mech. 424, 101-125.

MeI, C. C. 1985 Resonant reflection of surface water waves by periodic sandbars. J. Fluid Mech. 152, 315-335.

Mei, C. C., Hara, T. \& Naciri, M. 1988 Note on Bragg scattering of water waves by parallel bars on the seabed. J. Fluid Mech. 186, 147-162.

Mei, C. C., Stiassnie, M. \& Yue, D. K.-P. 2005 Theory and Applications of Ocean Surface Waves. Part 1. Linear Aspects. World Scientific.

Newman, J. N. 1975 Interaction of waves with two-dimensional obstacles: a relation between the radiation and scattering problems. J. Fluid Mech. 71, 273-282.

O'LeARY, M. 1985 Radiation and scattering of surface waves by a group of submerged, horizontal, circular cylinders. Appl. Ocean Res. 7, 51-57.

Porter, R. \& Evans, D. V. 1998 The trapping of surface waves by multiple submerged horizontal cylinders. J. Engng Math. 34, 417-433.

Porter, R. \& Evans, D. V. 2006 Scattering of flexural waves by multiple narrow cracks in ice sheets floating on water. Wave Motion 43, 425-443.

Porter, R. \& Porter, D. 2003 Scattered and free waves over periodic beds. J. Fluid Mech. 483, $129-163$.

SCHNUTE, J. T. 1967 Scattering of surface waves by submerged circular cylinders. Part II. Scattering by an infinite array of cylinders. Tech. Rep. 11. Department of Mathematics, Stanford University.

SChnute, J. T. 1971 The scattering of surface waves by two submerged cylinders. Proc. Camb. Phil. Soc. 69, 201-215.

SHEN, Y. \& ZHENG, Y. 2007 Interaction of oblique waves with an array of long horizontal circular cylinders. Sci. China E 50 (4), 490-509.

Thorne, R. C. 1953 Multipole expansions in the theory of surface waves. Proc. Camb. Phil. Soc. 49, 707-716. 
UrSELl, F. 1950 Surface waves on deep water in the presence of a submerged circular cylinder. Part I. Proc. Camb. Phil. Soc. 46, 141-152.

Ursell, F. 1951 Trapping modes in the theory of surface waves. Proc. Camb. Phil. Soc. 47, 347-358.

YANG, S., Wu, F., Zhong, H. \& Zhong, L. 2006 Large band gaps of water waves through twodimensional periodic topography. Phys. Lett. A 352, 426-430. 